\title{
Detection of amplified DNA sequences by reverse chromosome painting using genomic tumor DNA as probe
}

\author{
Stefan Joos ${ }^{1}$, Harry Scherthan², Michael R. Speicher ${ }^{2}$, Jürgen Schlegel $^{3}$, Thomas Cremer ${ }^{2}$, Peter Lichter \\ ' Angewandte Tumorvirologie, Deutsches Krebsforschungszentrum, W-6900 Heidelberg, Germany \\ ${ }^{2}$ Institut für Humangenetik und Anthropologie, Universität Heidelberg, W-6900 Heidelberg, Germany \\ ${ }^{3}$ Abteilung für Neuropathologie, Institut für Pathologie, Universität Heidelberg, W-6900 Heidelberg, Germany
}

Received: 30 October 1992

\begin{abstract}
A modification of "reverse chromosome painting" was carried out using genomic DNA from tumor cells as a complex probe for chromosomal in situ suppression hybridization to normal metaphase chromsome spreads. Amplified DNA sequences contained in such probes showed specific signals, revealing the normal chromosome positions from which these sequences were derived. As a model system, genomic DNAs were analyzed from three tumor cell lines with amplification units including the proto-oncogene c-myc. The smallest amplification unit was about $90 \mathrm{~kb}$ and was present in 16-24 copies; the largest unit was bigger than $600 \mathrm{~kb}$ and was present in 16-32 copies. Specific signals that co-localized with a differently labeled c-myc probe on chromosome band 8q24 were obtained with genomic DNA from each cell line. In further experiments, genomic DNA derived from primary tumor material was used in the case of a male patient with glioblastoma multiforme (GBM). Southern blot analysis using an epidermal growth factor receptor gene (EGFR) probe that maps to $7 \mathrm{p} 13$ indicated the amplification of sequences from this gene. Using reverse chromosome painting, signals were found both on band $7 \mathrm{pl} 3$ and bands 12q13-q15. Notably, the signal on 12q13-q15 was consistently stronger. The weaker $7 \mathrm{p} 13$ signal showed co-localization with the major signal of the differently labeled EGFR probe. A minor signal of this probe was seen on 12q13, suggesting cross-hybridization to ERB3 sequences homologous to EGFR. The results indicate co-amplification of sequences from bands $12 \mathrm{q} 13-\mathrm{q} 15$, in addition to sequences from band $7 \mathrm{p} 13$. Several oncogenes map to $12 \mathrm{q} 13-\mathrm{q} 15$ providing candidate genes for a tumor-associated proto-oncogene amplification. Although the nature of the amplified sequences needs to be clarified, this experiment demonstrates the potential of reverse chromosome painting with genomic tumor DNA for rapidly mapping the normal chromosomal localization of the DNA from which the amplified sequences were derived. In addition, a weaker staining of chromosomes 10 and $\mathrm{X}$ was consistently observed indicating that these chromosomes
\end{abstract}

Correspondence to: P. Lichter, Angewandte Tumorvirologie, 6. OG, Deutsches Krebsforschungszentrum, Im Neuenheimer Feld 280, W-6900 Heidelberg, Germany were present in only one copy in the GBM genome. This rapid approach can be used to analyze cases where no metaphase spreads from the tumor material are available. It does not require any preknowledge of amplified sequences and can be applied to screen large numbers of tumors.

\section{Introduction}

Following the discovery of amplified genes in cultured cells selected for drug resistance or responding to environmental stress, specific amplification of genomic sequences has also been discovered in numerous tumors. Amplified sequences in tumor genomes frequently contain specific proto-oncogenes (Alitalo and Schwab 1986). Cytogenetic analysis of tumor cell cultures has revealed that amplified DNA can be present either in double minute chromosomes (DMINs), small acentric chromosome-like structures that are frequently paired, or in homogeneously staining chromosomal regions (HSRs), visible with intermediate intensity using conventional banding techniques. However, our knowledge of the complexities of amplified sequences in primary tumors is still very limited.

Since the chromosomal origin of sequences in DMINs and HSRs cannot be determined by the analysis of banded chromosomes, assessment of the amplified genes is usually carried out by in situ and Southern blot hybridization of large numbers of proto-oncogene probes. However, these analyses rely entirely on the availability of suitable probe panels. In addition, co-amplified regions might often not be identified.

In the case of in situ hybridization, high quality metaphase spreads are required from the cells to be analyzed. However, in many cases (as in certain hematological and many solid tumors) such metaphases cannot be obtained. The possibilities of carrying out such an analysis on interphase nuclei (BarAm et al. 1992) remain restricted.

In the present study, an alternative approach is applied to analyze unknown amplified regions of a genome (Kallioniemi et al. 1992). This approach provides a new example of a strategy termed "reverse chromosome painting" (Carter et 


\section{Reverse Chromosome Painting}

\begin{abstract}
Labeled total DNA from source
(examples see below) to analyze
\end{abstract}
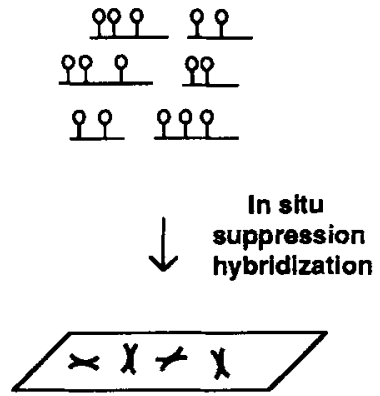

Normal chromosomes

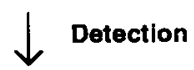

DNA from: results in Signal on normal chromosomes: (partial karyotype)

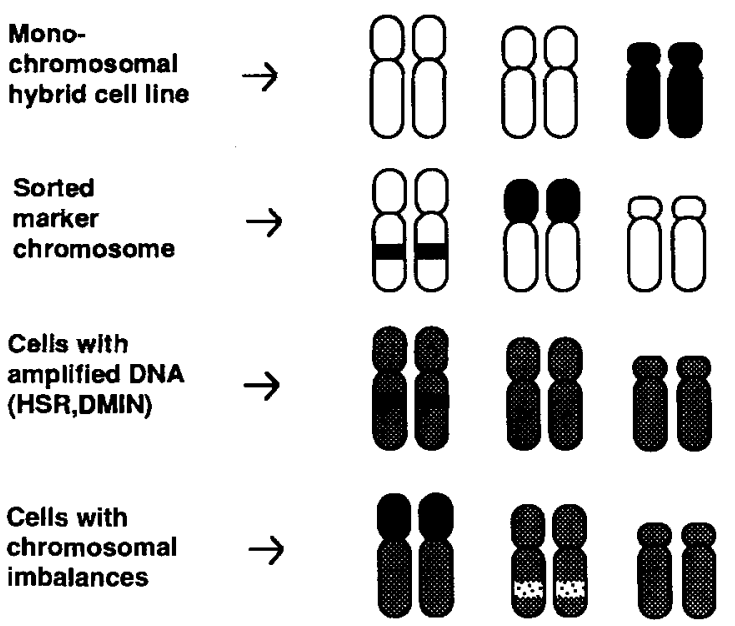

Fig. 1. Reverse chromosome painting. Schematic illustration of the reverse chromosome painting approach and several of its applications. Labeled DNA from various sources is hybridized to metaphase chromosome spreads from normal cells. The lower part of the scheme illustrates the results for four different DNA sources. Left column List of the DNA sources; right column partial karyotypes of the hybridized normal chromosome complements with the resulting hybridization signals. Unlabeled chromosomes or chromosomal regions are shown in white and strongly labeled regions are shown in black. Total genomic DNA as a probe (for example, tumor cell DNA from the same species), results in general chromosomal staining shown in grey. A staining stronger than general chromosomal staining due to over-representation of sequences is indicated in black, and staining weaker than general chromosomal staining due to under-representation of sequences is dotted. HSR Homogeneously staining chromosomal region; DMIN double minute

al. 1992). The principles of this procedure are outlined schematically in Fig. 1. The first applications of this approach were presented in studies investigating the chromosomal content of interspecies hybrid cells (Boyle et al. 1990; Kievits et al. 1990). The whole genomic DNA of a hybrid cell line was used as a probe for chromosomal in situ suppression (CISS) hybridization on metaphase spreads from normal cells of a species of interest. By this approach, all chromosomes and chromosomal segments of this species present in the hybrid cell were easily identified. Further improvements of this approach were achieved by species-specific polymerase chain reaction ( $\mathrm{PCR}$ ) amplification products from such hybrid lines (Lengauer et al. 1990; Lichter et al. 1990). Reverse chromosome painting has also been successfully used to identify marker chromosomes in disease cases. After flow sorting of a marker chromosome, its DNA was amplified and used as a probe for CISS hybridization to normal human chromosomes (Telenius et al. 1992). This allows the rapid identification of the origin(s) of the marker chromosome. Reverse chromosome painting also allows a comprehensive analysis for identifying imbalanced chromosomal material of entire genomes when using the whole genomic DNA as a probe (see also Kallioniemi et al. 1992 and the accompanying paper by du Manoir et al. 1993).

In the present study, we demonstrate the application of reverse chromosome painting to identify the chromosomal origin of amplified regions using genomic tumor cell DNA as a probe. First, three cell lines were analyzed with amplification units containing the proto-oncogene c-myc. Secondly, a primary glioblastoma multiforme (GBM) tumor was investigated, in which Southern blot analysis suggested multiple copies of the epidermal growth factor receptor (EGFR) gene.

\section{Materials and methods}

\section{Tumor material}

The cell lines Colo320-HSR and HL-60 were both obtained from the American Type Culture Collection. Colo320-HSR was derived from a colon tumor (Alitalo et al. 1983) and HL-60 from a promyelocytic leukemia (Collins and Groudine 1982; Dalla Favera et al. 1982). The cell line NCI-H60 was established from a small cell lung cancer (Little et al. 1983). Tumor 978/90 was derived from a male patient with GBM according to the World Health Organization (WHO) classification (Zülch 1979). Diagnosis was based on histological examination of paraffin-embedded tissue sections and confirmed on tissue that was frozen, and finally stained with hematoxylin-eosin.

\section{Preparation of tumor DNA}

Cell lines Colo320-HSR and HL60 were propagated in RPMI1640 supplemented with $10 \%$ fetal calf serum. Tissue samples of the glioblastoma tumor $978 / 90$ were frozen in liquid nitrogen immediately after surgery and then stored at $-80^{\circ} \mathrm{C}$. Genomic DNA was prepared by standard methods (Maniatis et al. 1982) applying proteinase K digestion, phenol and chloroform/isoamylalcohol (24:1) extraction, and was finally precipitated with ethanol. DNA from cell line NCI-H60 was kindly provided by J. D. Minna, NIH, Bethesda, Md.

\section{Cloned probes}

Cosmid cos-myc 72 containing an insert of about $30 \mathrm{~kb}$ from the c-myc gene (MYC) was originally isolated from a cosmid library of the Burkitt's lymphoma cell line IARC/BL72 (Joos et al. 1992). For detection of the EGFR gene (c-erb-1), a c-DNA clone termed pECE-EGFR (3.8kb Sall-Xhol fragment, Haley et al. 1987) was available. This clone spans almost the whole transcription unit, except for the last exon.

\section{In situ hybridization}

Metaphase chromosomes of normal blood lymphocytes $(46, \mathrm{XY})$ were prepared according to standard protocols of colcemid arrest, hypotonic 
treatment and methanol/acetic acid (3:1) fixation. The protocol used for in situ hybridization is described in detail in Lichter and Cremor (1992). DNA was labeled by nick translation in the presence of either digoxigenin-11-dUTP or biotin-11-dUTP. For a 12- $\mu 1$ hybridization volume, 0.3-1 $\mu \mathrm{g}$ biotin-labeled or digoxigenin-labeled tumor DNA was combined with $50 \mu \mathrm{g}$ Cotl fraction of human DNA. When tumor DNA was co-hybridized with individual cloned probes, $60 \mathrm{ng}$ digoxigenin-labeled cos-myc 72 or $200 \mathrm{ng}$ biotin-labeled pECE-EGFR was added to the labeled tumor DNA. Denatured probe was pre-annealed for $15 \mathrm{~min}$ at $37^{\circ} \mathrm{C}$ and hybridized to denatured chromosomes for $24 \mathrm{~h}$. Following post hybridization washes to a final stringency of $0.1 \times \mathrm{SSC}$ at $60^{\circ} \mathrm{C}$, hybridized DNA was detected via fluorescein isothiocyanate (FITC)conjugated avidin and anti-digoxigenin rhodamine. Hybridization signals of pECE-EGFR were amplified according to Pinkel et al. (1986). Chromosomes were counterstained with 4,6-diamino-2-phenyl-indole (DAPI). Slides were inspected directly using a conventional epifluorescence microscope and signals were counted to define hybridization efficiency. An additional analysis was performed using digitized images of FITC, rhodamine or DAPI fluroescence acquired with a cooled chargedcouple device (CCD) camera (Photometrics, Tuscon, Ariz.). For documentation, images of different fluorochromes from the same object were overlaid electronically following careful realignment using the software package Gene Join (Yale University) as described (Ried el al. 1992). Prior to overlaying, thresholding was applied to images obtained from the genomic DNA hybridization in order to show only the peak fluorescence. Photographs of digitized images were taken directly from the monitor.

\section{Results}

\section{Detection of amplified $8 q 24$ sequences containing c-myc}

The reverse chromosome painting technique was first applied for the detection of amplified sequences by using DNA from three tumor cell lines with a known amplification of the proto-oncogene c-myc. As listed in Table 1, the c-myc amplification unit in Colo320-HSR, HL60 and NCI-H60 differes with respect to length and copy number. All tumor DNAs labeled the whole set of normal chromosomes. Above this general staining, highly specific fluorescence signals on $8 \mathrm{q} 24$ were obtained with each of the three tumor DNAs (see Table 1). One example is presented in Fig. 2A, B. The signal specificity was independent of the fluorochrome used for detection. Of the evaluated metaphases, $80 \%-95 \%$ showed intense fluorescence signals on $8 \mathrm{q} 24(59 \%-84 \%$ on both homologs).

Table 1. Length and copy number of c-myc-containing amplification units compared with the efficiency of detecting the corresponding region by reverse painting

\begin{tabular}{llll}
\hline Cell line & Colo320-HSR & NCI-H60d.e & HL-60 \\
\hline Copy number & $16-32$ & 38 & $16-24$ \\
Size of amplicon & $>600 \mathrm{~kb}$ & $>340 \mathrm{~kb}$ & $\geq 90 \mathrm{~kb}$ \\
Evaluated metaphases & 75 & 46 & 44 \\
Metaphases with signats on $8 \mathrm{q} 24:$ & & \\
0 signals (\%) & 5.3 & 10.9 & 20.5 \\
1 signal (\%) & 10.7 & 13.0 & 20.5 \\
2 signals (\%) & 84.0 & 76.1 & 59.0 \\
\hline
\end{tabular}

\footnotetext{
a Alitalo et al. 1983

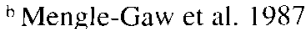

' Henglein et al. 1989

“Joos et al. 1992

'Little et al. 1983

${ }^{\mathrm{t}}$ Kienzler et al. 1986
}

In CCD images, a threshold analysis confirmed that the peak fluorescence intensities were always observed over band $8 \mathrm{q} 24$. Other signals of corresponding intensity were rarely observed and were considered to be background, since they did not appear consistently on the same chromosome region. Two signals could be seen in most interphase nuclei.

Co-hybridization experiments were carried out with cosmyc 72 as a probe to test further whether the signals observed after reverse chromosome painting of genomic DNA from the three cell lines were generated by amplified sequences containing $\mathrm{c}-\mathrm{myc}$. These experiments demonstrated that the signals from tumor and cosmid DNA were co-localized in all metaphase spreads analyzed from the three tumor cell lines. One example is shown in Fig. 2B.

\section{Amplification of two genomic regions containing proto-oncogenes in a glioblastoma tumor}

To test the potential of reverse chromosome painting for the detection of amplified DNA sequences in primary tumor material, we performed an analysis with DNA from a case of GBM. Southern blot analysis of this case indicated an amplification of the EGFR gene (previous symbol: ERBB; formerly avian erythroblastic leukemia viral ( $v$-erb-b) oncogene homolog) located on chromosome 7pl3. Reverse chromosome painting using the tumor DNA as probe revealed highly intense hybridization signals on 12q13-q15 (see Fig. 2C-E). In addition, a weaker signal was seen on 7p12-p13, consistently above the general chromosome staining. Both signals could be easily identified by conventional microscopy. Their specificity was also confirmed by a threshold analysis of CCD images as described above. A total of 32 digitized images was analyzed: the chromosome 12 signal was found on both homologs in $100 \%$ of the metaphase spreads, whereas $12.5 \%$ exhibited the signal on one and $87.5 \%$ on both chromosome 7 homologs. Unexpectedly, the signal on $12 q$ was much stronger compared with that on $7 \mathrm{p}$ (see Fig. 2C). Analysis of elongated chromosomes revealed that it is composed of two spatially separated signals, a highly intense signal on 12q14-15, and a proximal weaker signal, located most probably on $12 \mathrm{q} 13$ (see Fig. 2E). Interestingly the two latbeled regions $7 \mathrm{pl} 3$ and $12 q 13$ carry homologous sequences (see Discussion).

Using an EGFR c-DNA as a co-hybridization probe, the corresponding signal was found to co-localize with the tumor DNA signal on 7p (Fig. 2D) in all metaphase spreads analyzed $(n=32)$. In addition, a second, much weaker signal was found to co-localize with the major tumor DNA signal on band $12 q 13-q 15$. This minor signal was seen in $15 \%$ of the metaphase spreads on both chromosomes 12 , and in $37 \%$ on one chromosome 12 homolog. Thus, both the tumor DNA and the EGFR c-DNA probe generated two signals at the same chromosomal sites. However, the major signal obtained with the tumor DNA was on $12 \mathrm{q}$, whereas the major signal obtained with the c-DNA was on $7 p$.

Visual inspection of CCD images by several independent observers of normal metaphase spreads painted with the glioblastoma tumor DNA consistently revealed a weaker staining of both chromosomes 10 and the $\mathrm{X}$ chromosome (Fig. 2C; for details of the evaluation procedure, see du Manoir et al. 1993). This observation can be attributed to a 

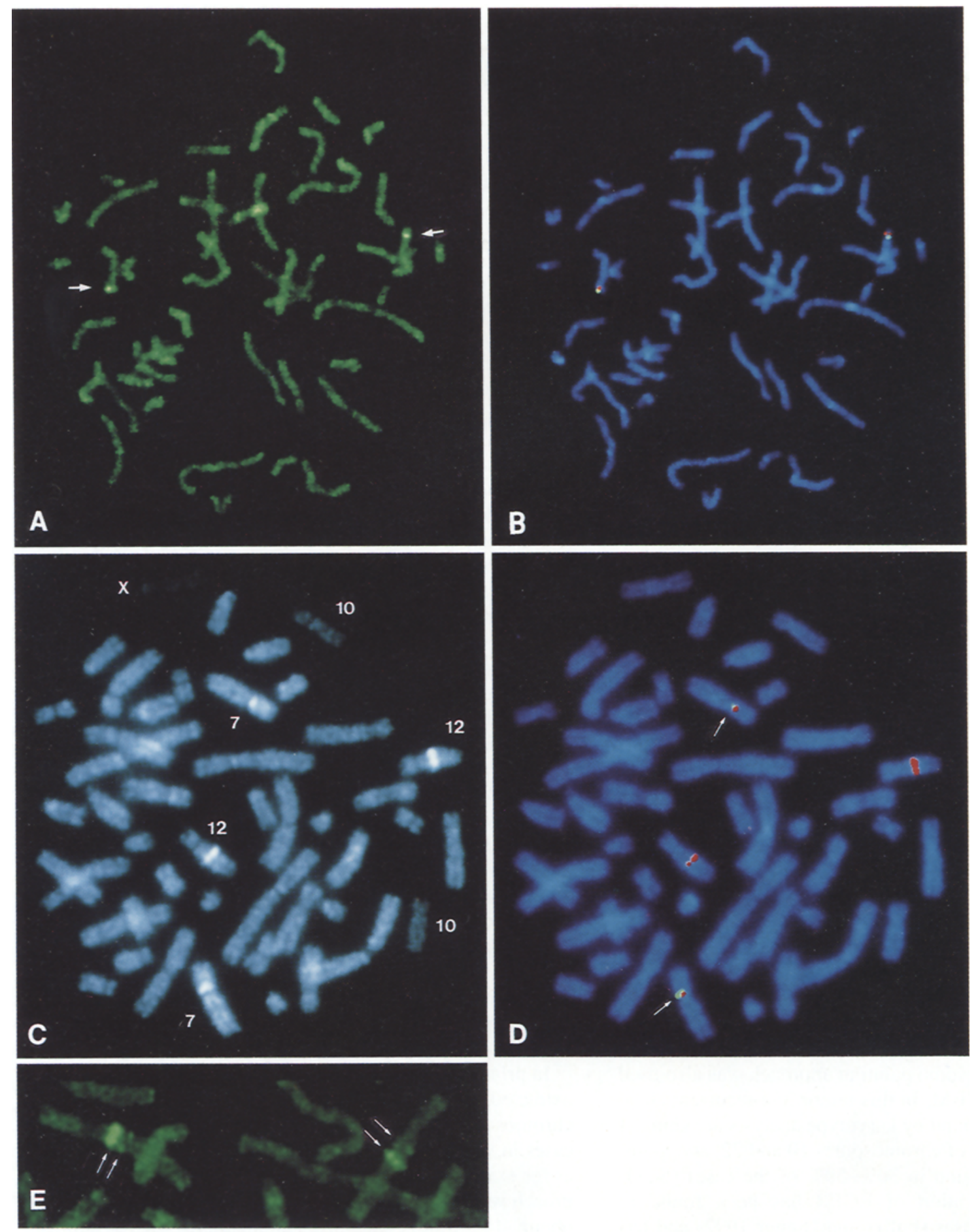

Fig. 2A-E. Reverse chromosome painting of normal chromosomes with a genomic tumor DNA. A. CCD image of a normal human metaphase spread, $46(\mathrm{XY})$, after CISS hybridization with biotin-labeled genomic DNA of the tumor cell line HL60. The hybridized probe was detected via FITC. Above the general level of chromosome painting, two highly intense signals are visible on chromosome 8q24 (arrows). B The same metaphase spread after overlaying of three digitized images each obtained from a different fluorochrome: (1) DAPI-stained chromosomes shown in blue; (2) the FITC image presented in $\mathbf{A}$ after a thresholding procedure to delineate only the peak fluorescence intensities, and showing green spots demonstrating the location of the most intense signals on 8q24; (3) digoxigenin-labeled probe for c-myc detected via rhodamine (rod spots). For illustration purposes, the image of the c-myc probe was shifted by two pixels to resolve the green and red signals. C Gray level CCD image of a normal metaphase chromosome spread hybridized with genomic DNA from a glioblastoma (GBM) carrying an amplification of the EGFR gene on chromosome 7p13. Digoxigenin-la-

beled tumor DNA was detected via rhodamine. Specific signals with high fluorescent intensity appear on the short arm of chromosome 7 (7p13) and, even stronger, on the long arm of chromosome 12 $(12 q 13-q 15)$. Note the weak labeling of the X chromosome and both chromosome 10 homologs suggesting an under-representation of these chromosomes in the tumor. D The same metaphase as in $\mathbf{C}$ presented after overlaying of three pseudocolored digitized images as described in B. DAPI-banded chromosomes are shown in blue, the rhodamine image of $\mathrm{C}$ (optimized to show the peak fluorescence) is seen in red. A co-hybridized EGFR probe, biotin-labeled and detected via FITC, is shown in green (arrows). Note the co-localization of the EGFR signal and the tumor DNA signal on 7 p13. E Partial prometaphase chromosome spread showing the hybridization signals on chromosome 12 obtained with biotinylated GBM DNA. Note that two signals (arrows) can be detected, a proximal weaker signal (most probably on 12q13) and a distal, much stronger signal (12q14-q15) 
loss of chromosome 10 and the hemizygosity of the $\mathrm{X}$ chromosome in the tumor genome derived from a male patient (see Discussion).

\section{Discussion}

In this study, we have demonstrated that amplified sequences can be readily detected by in situ hybridization of total genomic DNA to normal human chromosomes. The corresponding signals are immediately visible in the microscope. Reverse chromosome painting was carried out using the DNA of three tumor cell lines in which the proto-oncogene MYC is known to be amplified. The efficiency in visualizing chromosomal band 8q24 harboring MYC roughly correlated to the amount of sequences over-represented in the probe DNA. The cell line Colo-320 containing most of the additional sequences (16-32 copies of an amplification unit of more than $600 \mathrm{~kb}$ ) showed the highest efficiency (95\%) in delineating the corresponding region on one or both homologs (see Table 1). The lowest efficiency $(80 \%)$ was seen with cell line HL-60, which contains the smallest amount of multiplied DNA (approximately 20 copies of about $90 \mathrm{~kb}$ ). Thus, the sensitivity of the approach is at least in the range of detecting a 20-fold multiplication of a fragment approximately $100 \mathrm{~kb}$ in length. By analogy to gene mapping experiments, it is expected that signals on $30 \%-40 \%$ of the metaphases are sufficient to define a specifically labeled region. Therefore, the sensitivity defined by HL-60 in this report is probably an under-estimation of the general sensitivity of the approach. It should be noted that the typical length of amplification units described so far is between 200 and $2000 \mathrm{~kb}$ (Ruppert et al. 1991). This range includes the c-myccontaining units of the cell lines Colo320-HSR and NCI-H60 investigated in this study.

In addition to the highly intense signals on $8 \mathrm{q} 24$, the fluorescence along all chromosomes appeared not to be evenly distributed. The impact of such a distribution on the diagnosis of gains and losses of chromosomal regions is discussed below and analyzed in detail in du Manoir et al. (1992).

The reverse chromosome painting approach was also used to analyze a case of GBM. In this tumor, the recurrent chromosome aberrations found by karyotype analysis are gains of chromosome 7, losses of chromosomes 10 and 22, and gonosomes. DMINs are found in 30\%-50\% of the cases (Sandberg 1990). Amplification of EGFR has been found in $29 \%-50 \%$ of GBM cases (Fuller and Bigner 1992) and has also been detected by Southern blot analysis in the case analyzed in this study. This finding could be confirmed by reverse chromosome painting using the tumor DNA; a specific signal on band $7 \mathrm{p} 13$ co-localized with an EGFR hybridization signal. The additional split signal on the long arm of chromosome 12 was unexpected. The weaker signal on $12 q 13$ could be explained by cross-hybridization of the EGFR sequences contained in the tumor DNA to ERBB3, a homolog of the EGFR gene family mapping to $12 \mathrm{q} 13$. Coamplification of the ERB3 region can be considered as an alternative explanation.

The major signal on chromosome 12q14-q15 obtained with the GBM tumor DNA was much stronger than the signal on $7 \mathrm{p} 13$. Since the Southern blot data independently revealed an amplification of EGFR, the signal on 7p13 cannot be completely explained by a cross-hybridization of amplified $12 q$ sequences. Thus, co-amplification of sequences in both chromosomal regions is the most probable explanation for our findings. Notably, numerous genes that are located within $12 q 13-q 15$ have been found to be involved in tumorigenesis (Craig and McBride 1991), including the glioma-associated oncogene homolog (GLI), a member of the Krüppel family of zinc finger protein genes. This gene maps to $12 \mathrm{q} 13-\mathrm{ql} 4$ and has been found to be amplified in about $2 \%-4 \%$ of glioblastomas (Fuller and Bigner 1992). Further proto-oncogenes that might be contained in the amplified or co-amplified regions are the ras homolog RAP1B (12q14), the melanomaassociated antigen CD63 gene (12q12-q13), the retinoic acid receptor gene RAR-gamma (RARG on 12q 13), and the int-1 proto-oncogene (WNT1, former INT1, on 12q13). Thus, the results obtained by reverse chromosome painting have rapidly provided a series of candidate genes that might be amplified and that may possibly play a pathogenetic role in this tumor.

Until now, analysis of the material constituting DMINs relied on hybridization experiments of candidate sequences. As a rule, large batteries of proto-oncogene probes were used in time-consuming analyses. Furthermore, sequences present in DMINs and HSRs could often not be identified, because of the limited number of suitable probes. In contrast, the approach of reverse chromosome painting gives an immediate clue regarding the chromosomal origin of amplified genomic sequences. Conventional analyses have often revealed only one amplified sequence in DMINs. However, since DMINs in tumors can result from co-amplification of sequences from different areas of the genome (Alitalo and Schwab 1986), important information about amplified DNA might be missed. As demonstrated in this study, reverse chromosome painting allows us to visualize co-amplified chromosomal regions in a single experiment. Furthermore, this approach provides a tool for screening large numbers of cases with respect to chromosomal regions that are commonly amplified in a particular type of tumor, and also for studying the time course of amplification events during tumor development.

In principle, any cellular material from which DNA can be extracted can be used for this analysis. Thus, the reverse chromosome painting technique is particularly useful for cases in which the preparation of chromosome spreads fails, as for example in many solid tumors. The potential of the approach will be further increased if DNA from tissue sections obtained for routine diagnostic applications, such as paraffinembeded tissues, can be used as a probe. This would also allow the use of archived material for retrospective studies of tumor-associated chromosomal amplifications and other genetic imbalancies. With the advent of techniques for the general amplification of DNA by PCR using universal primers (e.g. degenerated oligonucleotide primed (DOP)-PCR, see Telenius et al. 1992), it can be expected that even small tissue samples will be sufficient as source material.

Inspection of the signal intensities along chromosomes not only revealed stronger signals indicative of amplification, but also showed areas of weaker staining. With the GBM DNA, chromosomes 10 and $\mathrm{X}$ were consistently stained less intensely than the average painted chromosomes. Loss of chromosome 10 copies is found in $60 \%$ of all GBM cases (Bigner 
and Vogelstein 1990). Thus, the weak labeling of chromosome 10 may indicate monosomy 10 in this tumor case demonstrating the detection of chromosome losses by reverse chromosome painting. Detailed analysis for accurately assessing such chromosomal gains and losses, including gonosomal hybridization patterns, is presented in du Manoir et al. (1993).

Acknowledgements. We thank Marika Kiessling (University of Heidelberg) for providing glioblastoma tumor material, and Gabi Stumm and Anette Merdes for technical help. This study was supported by the Verein zur Förderung der Krebsforschung in Deutschland, a grant from the European Community (PL 910031) and the Deutsche Krebshilfe.

\section{References}

Alitalo K, Schwab M (1986) Oncogene amplification in tumor cells. In: Klein G, Weinhouse $S$ (eds) Advances in cancer research. Academic Press, Orlando, pp 235-281

Alitalo K, Schwab M, Lin CC, Varmus HE, Bishop JM (1983) Homogeneously staining chromosomal regions contain amplified copies of an abundantly expressed cellular oncogene (c-myc) in malignant neuroendocrine cells from a human colon carcinoma. Proc Natl Acad Sci USA 80:1707-1711

Bar-Am I, Mor O, Yeger H, Shiloh Y, Avivi L (1992) Detection of amplified DNA sequences in human tumor cell lines by fluorescence in situ hybridization. Genes Chromosomes Cancer 4:314-320

Bigner SH, Vogelstein B (1990) Cytogenetics and molecular genetics of malignant gliomas and medulloblastoma. Brain Pathol 1:12-18

Boyle AL, Lichter P, Ward DC (1990) Rapid analysis of mouse-hamster hybrid cell lines by in situ hybridization. Genomics 7:127-130

Carter NP, Ferguson-Smith MA, Pemyman MT, Telenius H, Palmear AH, Leversha MA, Glancy MT, Wood SL, Cook K, Dyson HM, Ferguson-Smith ME, Willatt R (1992) Reverse chromosome painting: a method for the rapid analysis of aberrant chromosomes in clinical cytogenetics. J Med Genet 29:299-307

Collins S, Groudine M (1982) Amplification of endogeneous myc-related DNA sequences in a human myeloid leukaemia cell line. Nature 298:679-681

Craig IW, McBride OW (1991) Report of the committee on the genetic constitution of chromosome 12. Cytogenet Cell Genet 58:555-579

Dalla Favera R, Wong-Staal F, Gallo RC (1982) Onc gene amplification in promyelocytic leukaemia cell line HL-60 and primary leukaemic cells of the same patient. Nature 299:61-63

Fuller GN, Bigner SH (1992) Amplified cellular oncogenes in neoplasms of the human nervous system. Mutat Res 276:299-306

Haley J, Kinchington D, Whittle N, Ullrich A, Waterfield MD (1987) The epidermal growth factor receptor gene. Oncogenes, genes and growth factors. Wiley, New York

Henglein B, Synovzik H, Groitl P, Bornkamm GW, Hartl P, Lipp M (1989) Three breakpoints of variant $\mathrm{t}(2 ; 8)$ translocations in Burkitt's lymphoma cells fall within a region 140 kilobases distal from c-myc. Mol Cell Biol 9:2105-2113

Joos S, Haluska FG, Falk MH, Henglein B, Harmeister H, Croce CM, Bornkamm GW (1992) Mapping chromosomal breakpoints of
Burkitt's $\mathrm{t}(8 ; 14)$ translocations far upstream of c-myc. Cancer Res $52: 6547-6552$

Kallioniemi O-P, Kallioniemi A, Chen L-C, Sudar D, Rutovitz D, Smith HS, Gray JW, Pinkel D, Waldman FW (1992) Many different chromosomal regions may undergo amplification during cancer initiation and progression (abstract). Am J Hum Genet 51:A40

Kievits T, Devilee P, Wiegant J, Wapenaar MC, Cornelisse CJ, van Ommen GJB, Pearson PL (1990) Direct nonradioactive in situ hybridization of somatic cell hybrid DNA to human lymphocyte chromosomes. Cytometry 11:105-109

Kinzler KW, Zehnbauer BA, Brodeur GM, Seeger RC, Trent JM, Meltzer PS, Vogelstein B (1986) Amplification units containing human N-myc and c-myc genes. Proc Natl Acad Sci USA 83:11571174

Lengauer C, Riethman H, Cremer T (1990) Painting of human chromosomes generated from hybrid cell lines by PCR with Alu and L1 primers. Hum Genet $86: 1-6$

Lichter P, Cremer T (1992) Chromosome analysis by non-isotopic in situ hybridization. In: Rooney DE, Czepulkowski BH (eds) Human cytogenetics. Vol I. Constitutional analysis. IRL, Oxford New York Tokyo, pp 157-192

Lichter P, Ledbetter SA, Ledbetter DH, Ward DC (1990) Fluorescence in situ hybridization with Alu and L1 polymerase chain reaction probes for rapid characterization of human chromosomes in hybrid cell lines. Proc Natl Acad Sci USA 87:6634-6638

Little CD, Nau MM, Carney DN, Gazdar AF, Minna JD (1983) Amplification and expression of the c-myc oncogene in human lung cancer cell lines. Nature 306: 194-196

Maniatis T, Fritsch EF, Sambrook J (1982) Molecular cloning: a laboratory manual. Cold Spring Harbor Laboratory, Cold Spring Harbor, NY

Manoir S du, Speicher MR, Joos S, Schröck E, Popp S, Döhner H, Kovacs G, Robert-Nicoud M, Lichter P, Cremer T (1993) Detection of complete and partial chromosome gains and losses by comparative genomic in situ hybridization. Hum Genet $90: 580-610$

Mengle-Gaw L, Rabbitts TH (1987) A human chromosome 8 region with abnormalities in B-cell, HTLV-1 T cell and myc amplified tumours. Embo J 6:1959-1965

Pinkel D, Gray JW, Trask B, Engh G van den, Fuscoe J, Dekken H van (1986) Cytogenetic analysis by in situ hybridization with fluorescently labeled nucleic acid probes. Cold Spring Harbour Symp Quant Biol 51:151-157

Ried T, Baldini A, Rand TC, Ward DC (1992) Simultaneous visualization of seven different DNA probes by in situ hybridization using combinatorial fluorescence and digital imaging microscopy. Proc Natl Acad Sci USA 89:1388-1392

Ruppert JM, Vogelstein B, Kinzler KW (1991) The zinc finger protein GLI transforms primary cells in cooperation with adenovirus. E1A. Mol Cell Biol 11:1724-1728

Sandberg AA (1990) The chromosomes in human cancer and leukemias. Elsevier, New York

Telenius H, Pelmear AH, Tunnacliffe A, Carter NP, Behmel A, Ferguson-Smith MA, Nordensköld M, Pfragner R, B.A.J P (1992) Cytogenetic analysis by chromosome painting using DOP-PCR amplified flow-sorted chromosomes. Genes Chromosomes Cancer 4:257-263

Zülch KJ (1979) International typing of tumors of the central nervous system. International classification of tumors. No 21. WHO, Geneva
Note added in proof. The following highly relevant article has appeared in print since submission of this paper: Kallioniemi A, Kallioniemi O-P, Sudar D, Rutovitz D, Gray JW, Waldman F, Pinkel D (1992) Comparative genomic hybridization for molecular cytogenetic analysis of solid tumors. Science $258: 818-821$ 